\title{
Superoxide flashes: illuminating new insights into cardiac ischemia and reperfusion injury
}
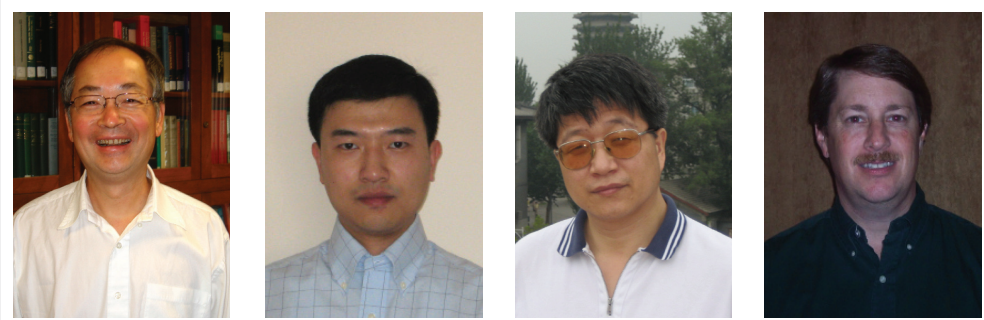

“...the dogma suggests that the opening of MPTP

is detrimental to the cell and MPTP activation is

widely thought to contribute to disease..."

\section{Shey-Shing Sheu', Wang Wang, Heping Cheng \& Robert T Dirksen}

†Author for correspondence: Departments of Pharmacology \& Physiology Anesthesiology and Medicine, Mitochondrial Research \& Innovation Group, University of Rochester Medical Center, 601 Elmwood Avenue, Rochester, NY 14642, USA = Tel.: +1 5852753381 = Fax: +1 5852732652

m.sheyshing_sheu@urmc.rochester.edu

Although the mitochondrial permeability transition pore (mPTP) was first discovered almost 30 years ago [1], it did not attract significant research attention until the 1990s when several studies implicated mPTP in apoptosis [2]. Today, the dogma suggests that the opening of $\mathrm{mPTP}$ is detrimental to the cell and mPTP activation is widely thought to contribute to disease in cancer, neurodegenerative diseases, stroke, muscular dystrophy and cardiac reperfusion injury [3]. Multiple factors including $\mathrm{Ca}^{2+}, \mathrm{OH}^{-}, \mathrm{P}_{\mathrm{i}}$, cyclophilin $\mathrm{D}$, reactive oxygen species (ROS) and reactive nitrogen species (RNS) trigger $\mathrm{mPTP}$ opening [4]. However, whether mPTP activation feeds back to alter mitochondrial ROS generation remains unclear. We recently demonstrated that under normal conditions, individual mitochondria undergo spontaneous transient bursts of quantal superoxide generation, termed 'superoxide flashes' [5]. Superoxide flashes are observed in all cell types investigated to date and are triggered by a surprising functional coupling between $\mathrm{mPTP}$ activation and electron transport chain (ETC)-dependent superoxide production. Additionally, reoxygenation following anoxia leads to uncontrolled superoxide flash genesis in cardiomyocytes. This positive feedback mechanism for $\mathrm{mPTP} / \mathrm{ETC}$-dependent ROS generation may drive localized redox signaling in individual mitochondria under physiological conditions, and when left unchecked, contributes to global cellular oxidative stress under pathological conditions in cardiac disease. The mPTP activity-dependent cell life and death determination imposes new challenges and opportunities in the pursuit of therapeutic agents for treating diseases in which oxidative stress has been implicated such as cardiac ischemia-reperfusion injury.

\section{Mitochondrial permeability transition pore as a drug target for attenuating reperfusion injury}

The crucial role of mPTP in causing cell death has put it as a new drug target for treating diseases across a wide spectrum of organs including the heart, liver, nervous system, lung and muscle, as well as cancer $[6,7]$. In a recent report, administration of the mPTP inhibitor cyclosporine A at the time of percutaneous coronary intervention in 30 patients was found to reduce infarct size greater than that observed with placebo [8]. These clinical data are consistent with numerous basic scientific studies demonstrating that mPTP activation is a key step in the pathogenesis of ischemia-reperfusion injury $[9,10]$.

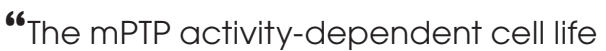
and death determination imposes new challenges and opportunities in the pursuit of therapeutic agents..."
}

The sequence of events in cardiac ischemiareperfusion injury begins with lactic acidosis of myocytes during ischemia. Cellular acidosis augments $\mathrm{Na}^{+} / \mathrm{H}^{+}$exchange activity to move $\mathrm{H}^{+}$out of cells, resulting in intracellular $\mathrm{Na}^{+}$

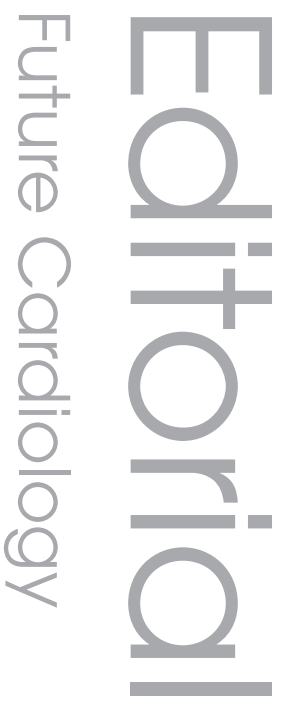


overload. Subsequently, the plasma membrane $\mathrm{Na}^{+} / \mathrm{Ca}^{2+}$ exchanger would operate less effectively in forward mode to transport $\mathrm{Ca}^{2+}$ out of the myocyte and more effectively to bring $\mathrm{Ca}^{2+}$ into the cell during conditions favoring reverse mode $\mathrm{Na}^{+} / \mathrm{Ca}^{2+}$ exchange, ultimately resulting in myoplasmic $\mathrm{Ca}^{2+}$ overload. The increase in myoplasmic $\mathrm{Ca}^{2+}$ is then taken up by mitochondria, resulting in a mitochondrial $\mathrm{Ca}^{2+}$ overload. During reperfusion, the reintroduction of normal oxygen and $\mathrm{H}^{+}$concentrations rapidly 'wakes up' the ETC, which leads to a massive increase in ROS generation. The combination of mitochondrial $\mathrm{Ca}^{2+}$ and ROS overload causes the opening of mPTP, release of cytochrome $c$ and apoptosis or necrosis $[11,10]$. Since one of the end points for ischemia-reperfusion injury is the opening of the mPTP, the ability of prior cyclosporine A administration to reduce infarct size is intuitively expected.

\section{Why other pharmacological agents failed in ischemia-reperfusion clinical trials}

Intriguingly, the aforementioned clinical study with a positive outcome is an exception rather than a norm. Over just the last decade, several clinical trials have been launched using pharmacological interventions designed to reduce acute myocardial infarction during reperfusion injury. The results of most of these studies either failed to show beneficial effects or are considered controversial [12]. These clinical trials include:

- $\mathrm{Ca}^{2+}$ channel inhibition using diltiazem and $\mathrm{MgSO}_{4}$ to decrease cellular $\mathrm{Ca}^{2+}$ overload;

- $\mathrm{Na}^{+} / \mathrm{H}^{+}$inhibitors, such as cariporide, to decrease cellular $\mathrm{Na}^{+}$overload;

- Anti-inflammatory and antioxidant agents such as fluosol and recombinant human superoxide dismutase to detoxify ROS;

- Pharmacological agents, such as adenosine, and volatile anesthetics to precondition the heart to better handle reperfusion insult.

The key reasons for the discrepancy between the preclinical and clinical studies have been discussed extensively in several recent reviews [12-14]. In many cases, patients in these clinical studies were older and suffering from other complications in addition to ischemic heart disease. As a result these patients were often taking multiple medications, with the pharmacokinetic parameters of each agent, including drug absorption, distribution and metabolism, being difficult to assess. Another factor that may limit the applicability of these studies is the lack of solid quantitative information regarding the therapeutic index of the drugs used. Therefore, it is likely that in some studies the dose of drug required to elicit a therapeutic response without unacceptable adverse effects was not achieved. Moreover, most of the drugs used in these studies were not targeted to a specific organ (e.g., heart) or a specific organelle (e.g., mitochondria).

\section{Coupling of MPTP to ROS generation}

Our recent findings of mPTP-coupled quantal ROS generation [5], provides new insight into why such treatments lead to divergent outcomes. The major finding of our study is the serendipitous discovery and subsequent characterization of novel transient superoxide producing superoxide flashes.

66...superoxide flashes result from an unexpected functional coupling between transient openings of the MPTP and ETC activity."

Superoxide flashes are brief bursts of superoxide production within the matrix of single mitochondria in intact quiescent cells. These superoxide flashes result from an unexpected functional coupling between transient openings of the MPTP and ETC activity. Superoxide flashes are found in a wide range of different cells including cardiac myocytes, neurons, neuroendocrine cells, skeletal muscle fibers and clonal cell lines. While the fundamental properties (magnitude and duration) of superoxide flashes are similar in all cells, flash frequency varies dramatically between cell types. In general, flash frequencies tend to be higher in dividing or proliferative cells and lower in terminally differentiated cells. Importantly, we found that superoxide flash frequency decreases during cardiac hypoxia or anoxia and exhibits a marked rebound increase during early reoxygenation after anoxia.

\section{Yin \& yang of superoxide flashes: physiological \& pathological significance}

Superoxide is the primary ROS produced by cells, which means that physiological and pathological ROS signaling begins with superoxide production. Thus, just as quantal localized calcium release events $\left(\mathrm{Ca}^{2+}\right.$ sparks) underlie intracellular calcium signaling in the 
heart [15], superoxide flashes may represent elementary events of ROS production and signaling within the mitochondrial matrix. Physiologically, these stochastic elementary events of mitochondrial superoxide production may regulate redox-sensitive processes that are restricted to either within or immediately adjacent to the flash-producing mitochondrion. Indeed, recent evidence indicates that an array of cellular functions (e.g., channel-transporter activity, $\mathrm{Ca}^{2+}$ spark production, kinase-phosphatase activity and gene expression) are highly redox sensitive [16]. Left uncontrolled, a high frequency of superoxide flash activity may lead to local, and eventually widespread, cellular damage and thus contribute to the pathogenesis of a variety of different oxidative stress-related disorders.

\section{New insights into future drug development in treating oxidative stress-mediated diseases}

It is well known that massive openings of the mPTP play a critical role in cell pathology. As demonstrated in our study, pathological permeability transition pore openings and uncontrolled superoxide flash activity contributes to the destructive bursts of ROS observed during the early phase of reperfusion. Superoxide flashes may also be of relevance to the long-standing debate regarding the proposed contribution of transient openings of the $\mathrm{MPTP}$ within a physiological context. Since superoxide flashes occur relatively infrequently under resting conditions in differentiated cells, superoxide flash activity under these conditions likely reflects infrequent basal mPTP activity that contributes to local physiological ROS signaling. Thus, it is conceivable that clinical trials of drugs designed to block ROS stress resulting from uncontrolled MPTP activity could be detrimental because these agents may also inhibit the physiological levels of superoxide generation required for local ROS signaling in healthy tissues. In addition, as the drugs used in these studies are not specifically targeted to mitochondria, the concentrations required to inhibit mitochondrial ROS production are high, and thus, confounded by unavoidable toxicity. Therefore, futurerelated drug development initiatives will need to identify agents that effectively block massive mPTP opening and uncontrolled ROS production, but not basal, physiologically-relevant ROS production. Moreover, since superoxide flashes are triggered by transient openings of the mPTP, superoxide flash activity may potentially be used as both a biomarker of
mPTP function or activity and as an index for screening potential therapeutic agents to protect from ischemia-reperfusion injury.

\section{Future perspective}

Our discovery of quantal superoxide production within the mitochondrial matrix will stimulate future studies of intracellular ROS signaling, mitochondrial functionality and regulation of cell metabolism. First, superoxide flashes constitute elementary events of transient and quantal ROS generation within a single mitochondrion. Previously, researchers believed that mitochondrial ROS production results solely as a minimal byproduct of continuous or constitutive mitochondrial respiration. Second, our discovery of intermittent mitochondrial superoxide producing events reflects a new paradigm of mitochondrial function in living cells, namely that mPTP activity accelerates or resets mitochondrial respiration and stimulates superoxide generation by the ETC. Consequently, mitochondrial metabolic rate may be regulated by external factors that influence mPTP opening. The exact signal(s) that triggers transient mPTP opening and subsequent quantal superoxide flash generation is unknown; an answer to this question will be of great importance since it may help to nail down factors that control cell metabolism, cell function and cell fate under both resting and stress conditions. For now, we believe that low levels of constitutive ROS production during continuous mitochondria respiration is a likely candidate.

\footnotetext{
"The next major leap in biomedicine

will reflect a shift in this paradigm to include energetic and redox defects."
}

Finally, at the cellular level, the temporal and spatial integration of quantal superoxide signals provides a mechanism for diversification of ROS signaling in various cell processes. As we have demonstrated in our study, the basal frequency of superoxide flash activity varies significantly between different cell types, which may indicate differences in cell metabolic activity, proliferation rate and functionality. For example, neonatal cardiac myocytes exhibit a much higher superoxide flash frequency than adult cardiac myocytes, consistent with higher metabolic and growth rates in developing myocytes. Taken together, the transient opening of mPTP serves as a code for both cellular redox signaling and metabolic coupling. As indicated in a recent paper by Wallace [17], historically, the etiology of human diseases is mostly attributed to structural (anatomical) and genetic 
(Mendelian) defects. However, this disease paradigm has come to a bottleneck. The next major leap in biomedicine will reflect a shift in this paradigm to include energetic and redox defects. Thus, we predict that elucidation of the mechanisms by which mitochondrial superoxide flashes encode elementary events of energy and ROS generation will greatly change the landscape of future biomedical research and drug development.

\section{Acknowledgements}

We thank Drs Paul Brookes and George Porter for their comments on our manuscript.
Financial \& competing interests disclosure

This work was supported by NIH grants HL33333 (to Shey-Shing Sheu), AR044657 (to Robert Dirksen), Chinese National Natural Science Foundation and Major State Basic Research Development Programs of China (to Heping Cheng). The authors have no other relevant affiliations or financial involvement with any organization or entity with a financial interest in or financial conflict with the subject matter or materials discussed in the manuscript apart from those disclosed.

No writing assistance was utilized in the production of this manuscript.

\section{Bibliography}

1. Hunter DR, Haworth RA: The $\mathrm{Ca}^{2+}$ induced membrane transition in mitochondria. I. The protective mechanisms. Arch. Biochem. Biophys. 195, 453-459 (1979).

2. Loeffler M, Kroemer G: The mitochondrion in cell death control: certainties and incognita. Exp. Cell. Res. 256, 19-26 (2000).

3. Rasola A, Bernardi P: The mitochondrial permeability transition pore and its involvement in cell death and in disease pathogenesis. Apoptosis 12, 815-833 (2007).

4. Leung AW, Halestrap AP: Recent progress in elucidating the molecular mechanism of the mitochondrial permeability transition pore. Biochim. Biophys. Acta 1777, 946-952 (2008).

5. Wang W, Fang H, Groom L et al:: Superoxide flashes in single mitochondria. Cell 134, 279-290 (2008).

6. Bernardi P, Krauskopf A, Basso E et al.: The mitochondrial permeability transition from in vitro artifact to disease target. FEBS J. 273 , 2077-2099 (2006).

7. Armstrong JS: Mitochondria: a target for cancer therapy. Br. J. Pharmacol. 147, 239-248 (2006)

8. Piot C, Croisille P, Staat P et al.: Effect of cyclosporine on reperfusion injury in acute myocardial infarction. N. Engl. J. Med. 359, 473-481 (2008).
9. Di Lisa F, Bernardi P: Mitochondria and ischemia-reperfusion injury of the heart: fixing a hole. Cardiovasc. Res. 70, 191-199 (2006).

10. Murphy E, Steenbergen C: Mechanisms underlying acute protection from cardiac ischemia-reperfusion injury. Physiol. Rev. 88, 581-609 (2008).

11. Brookes PS, Yoon Y, Robotham JL, Anders MW, Sheu SS: Calcium, ATP, and ROS: a mitochondrial love-hate triangle. Am. J. Physiol. Cell. Physiol. 287, C817-33 (2004).

12. Dirksen MT, Laarman GJ, Simoons ML, Duncker DJ: Reperfusion injury in humans: a review of clinical trials on reperfusion injury inhibitory strategies. Cardiovasc. Res. 74, 343-355 (2007).

13. Tissier R, Berdeaux A, Ghaleh B et al. Making the heart resistant to infarction: how can we further decrease infarct size? Front. Biosci. 13, 284-301 (2008).

14. Yellon DM, Hausenloy DJ: Myocardial reperfusion injury. N. Engl. J. Med. 357, 1121-1135 (2007).

15. Cheng H, Lederer WJ, Cannell MB: Calcium sparks: elementary events underlying excitation-contraction coupling in heart muscle. Science 262, 740-744 (1993).

16. Veal EA, Day AM, Morgan BA: Hydrogen peroxide sensing and signaling. Mol. Cell 26, 1-14 (2007).

17. Wallace DC: Mitochondria as chi. Genetics 179, 727-735 (2008).

\section{Affiliation}

- Shey-Shing Sheu

Departments of Pharmacology \&

Physiology, Anesthesiology, and Medicine,

Mitochondrial Research \& Innovation

Group, University of Rochester Medical

Center, 601 Elmwood Avenue, Rochester,

NY 14642, USA

Tel.: +1 5852753381

Fax: +15852732652

sheyshing_sheu@urmc.rochester.edu

- Wang Wang

Department of Integrative Biology \& Physiology, University of Minnesota Medical School, 6-125 Jackson Hall, 321

Church Street SE, Minneapolis,

MN 55455, USA

Tel.: +1 6126255902

Fax: +16126255149

wang1514@umn.edu

- Heping Cheng

Institute of Molecular Medicine \&

National Laboratory of Biomembrane \&

Membrane Biotechnology, Peking

University, Beijing 100871, China

Tel.: +8601062758383

Fax: +011 861062765954

chengp@pku.edu.cn

- Robert Dirksen

Department of Pharmacology \&

Physiology, University of Rochester

Medical Center, 601 Elmwood Avenue,

Rochester, NY 14642, USA

Tel.: +1 5852754824

Fax: +15852732652

robert_dirksen@urmc.rochester.edu 